\title{
RISK FACTORS FOR LEFT ATRIAL THROMBUS FROM TRANSESOPHAGEAL ECHOCARDIOGRAPHY FINDINGS IN ISCHEMIC STROKE PATIENTS
}

\author{
TOMOHIRO KUMAGAI ${ }^{12) 3)}$, YUTAKA MATSUURA ${ }^{1)}$, TEIJI YAMAMOTO ${ }^{2)}$, \\ YOSHIKAZU UGAWA ${ }^{2)}$ and TETSUHITO FUKUSHIMA ${ }^{3)}$ \\ ${ }^{1)}$ Division of Neurology, Ohta Nishinouchi Hospital, Koriyama, Japan, ${ }^{2}$ Department of Neurology, Fuku- \\ shima Medical University School of Medicine, Fukushima, Japan, ${ }^{3)}$ Department of Hygiene \& Preventive \\ Medicine, Fukushima Medical University School of Medicine, Fukushima, Japan
}

(Received June 28, 2013, accepted October 30, 2014)

\begin{abstract}
Background : To identify the cause of cerebral embolism, we performed transesophageal echocardiography (TEE) in patients suspected of embolic brain infarction including transient ischemic attack (TIA). We analyzed TEE findings and investigated factors associated with left atrial thrombus (LAT) detected by TEE.

Methods : We enrolled 98 consecutive patients who underwent TEE and had acute brain infarction or TIA that was possibly due to embolism. We assessed age, sex, presence of atrial fibrillation (AF), days from admission to TEE and TEE findings, including the prevalence of LAT, spontaneous echo contrast (SEC), left atrial appendage (LAA) slow flow velocity, patent foramen ovale (PFO), atrial septal aneurysm and aortic plaque (ASA).

Results : LAT was detected with TEE in 20 patients (20\%). The factors that were significantly associated with the presence of LAT were male sex (unadjusted odds ratio (OR), $3.94 ; 95 \%$ confidence interval (CI), 1.07-14.58; $p=0.037$ ), presence of AF (unadjusted OR, 9.58 ; 95\% CI, 2.58$35.50 ; p<0.001$ ), SEC (unadjusted OR, $8.48 ; 95 \% \mathrm{CI}, 2.57-28.00 ; p<0.001$ ) and LAA slow flow velocity (unadjusted OR, $5.18 ; 95 \% \mathrm{CI}, 1.59-16.91 ; p=0.005$ ). Multivariate logistic regression analysis revealed that male sex (adjusted OR, $5.30 ; 95 \% \mathrm{CI}, 1.09-25.71 ; p=0.039$ ), presence of AF (adjusted OR, 8.97 ; 95\% CI, 1.10-73.20 ; $p=0.041$ ) and SEC (adjusted OR, 10.87 ; 95\% CI, 1.001$118.0 ; p=0.049)$ were independently associated with LAT, but LAA slow flow velocity was not.

Conclusion : SEC is an important risk factor associated with LAT in patients suspected of embolic brain infarction that is independent of AF.
\end{abstract}

Key words : transesophageal echocardiography (TEE), left atrial thrombus (LAT), ischemic stroke

\section{INTRODUCTION}

Stroke is the major cause of mortality and the fourth leading cause of death in Japan ${ }^{1)}$ and the third leading cause of death in Western countries ${ }^{2}$. Ischemic stroke is the most common type of stroke in developed countries, and it can be further divided into several subtypes based on their size and the location of the affected cerebral arteries. In addition, ischemic strokes can be classified into three main subtypes based on their pathogenesis : lacunar, atherothrombotic, and cardioembolic ${ }^{3)}$.

A previous study demonstrated that the 5-year survival rate of cardioembolic infarction was the lowest among ischemic stroke subtypes ${ }^{4)}$. It is well known that atrial fibrillation (AF), which causes left atrial thrombus (LAT) that may produce an embolism, is a common cause of ischemic stroke, and that AF-related strokes are accompanied by severe neurological deficits, disability and high mortality ${ }^{5}$.

Corresponding author : Tomohiro Kumagai E-mail: kuma@fmu.ac.jp

https://www.jstage.jst.go.jp/browse/fms http://www.fmu.ac.jp/home/lib/F-igaku/ 
Transesophageal echocardiography (TEE) is superior to transthoracic echocardiography (TTE) in detecting high risk sources and potential sources of cerebral embolism, such as right-to-left shunt, LAT in the left atrial appendage (LAA), spontaneous echo contrast (SEC) in the left atrium and aortic plaque ${ }^{6)}$. To identify the cause of cerebral embolism, we performed TEE in 98 patients suspected of embolic brain infarction or transient ischemic attack (TIA).

In this study, we analyzed the TEE findings and investigated factors associated with LAT detected by TEE.

\section{METHODS}

We enrolled 98 consecutive patients suspected of embolic brain infarction or TIA who were admitted to Ohta Nishinouchi Hospital from April 2004 to February 2006. All of these patients underwent TEE to evaluate a possible embolic mechanism. We performed TEE after consent was obtained from the patient or a family member. All patients underwent brain computed tomography (CT) or magnetic resonance imagining (MRI), including diffusionweighted imaging, fluid-attenuated inversion recovery imaging, T2-weighted imaging and magnetic resonance angiography. Brain infarction was diagnosed as an acute neurological event lasting $\geq 24 \mathrm{~h}$ and associated with focal hyperintensity on MRI. TIA was defined as sudden onset of a focal neurological deficit or amaurosis fugax that lasted $<24 \mathrm{~h}$ and was suspected to be of cerebrovascular origin ${ }^{3)}$. We diagnosed AF from the patient's medical history or by identification on 12-lead electrocardiogram and carried out continuous electrocardiographic monitoring or 24-h Holter electrocardiogram to detect paroxysmal AF. We assessed age, sex, presence of chronic or paroxysmal AF, days from admission to TEE and TEE findings, including the prevalence of LAT, SEC, LAA slow flow velocity, patent foramen ovale (PFO), atrial septal aneurysm (ASA) and aortic plaque.
TEE was performed with patients fasting for at least 6 hours before the examination using an ACUSON Sequoia (Mochida-Siemens Medical Systems, Tokyo, Japan) with a 3.5-7 MHz wideband multiplane probe. Lidocaine spray was routinely used for local anesthesia, which covered the pharynx. We used 5-10 mg diazepam intravenously for sedation, if necessary. The patients were placed in the left lateral decubitus position during probe insertion. The probe was slowly advanced to the esophagus. A careful survey of the entire atrium, including the LAA, was performed, and the presence of SEC was confirmed by decreasing the gain settings to exclude background noise artifact. SEC was identified as a dynamic and slowly swirled smokelike signal. LAA slow flow velocity was defined as peak velocity $\leq 30$ $\mathrm{cm} / \mathrm{s}$. The LAA was closely inspected for the presence of thrombi using multiplane imaging from $0^{\circ}$ to $180^{\circ}$. PFO was assessed by injecting agitated saline and having the patient perform a Valsalva maneuver. The numbers of microbubbles with and without contrast agent were compared. PFO was diagnosed when microbubbles were visualized in the left atrium during the Valsalva maneuver. At the same time, ASA was diagnosed when the atrial septum extended $\geq 11 \mathrm{~mm}$ into the left or right atrium, or both. Aortic atheroma was defined as plaque thickness $\geq 4 \mathrm{~mm}$ (Fig. 1a, b). Two same neurologists, who knew clinical, laboratory and CT/MRI findings, performed TEE and analyzed all TEE data.

Chi-square and Mann-Whitney tests were used to compare variables between the two groups with and without LAT. Factors found to be significantly associated with outcome were also examined by multivariate logistic regression analysis. A $p$ value $<0.05$ was considered statistically significant for all analyses. All analyses were performed using Statistical Package for the Social Science for Windows software (SPSS version 17.0 ; SPSS, Chicago, IL, USA).

This study was approved by the Clinical Research Ethics Committee of Ohta Nishinouchi Hos-
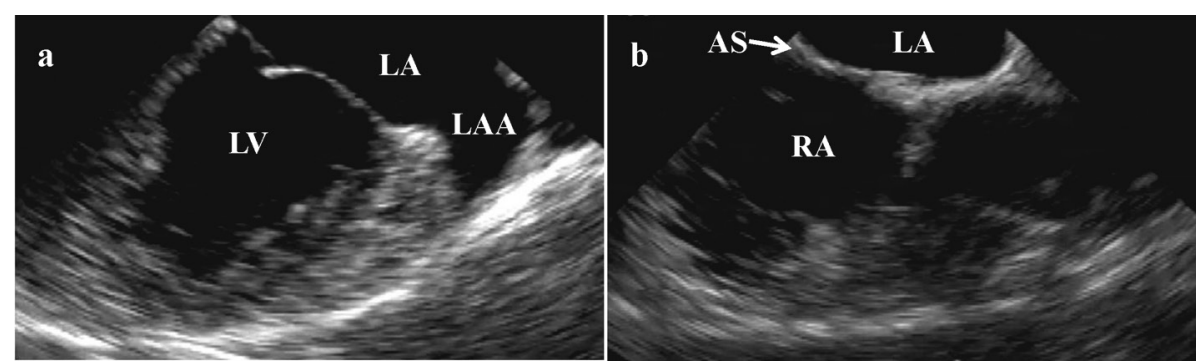

Fig. 1a, b. Survey areas by transesophageal echocardiography. LA, left atrium ; LAA, left atrial appendage ; LV, left ventricle ; RA, right atrium ; AS, atrial septum. 
pital on April 23, 2014, and the unlinkable anonymize data which were provided by this hospital were analyzed in this study.

\section{RESULTS}

Baseline clinical characteristics and TEE findings are summarized in Table 1 . Among the 98 patients enrolled in this study, 10 patients (10\%) had a TIA, 46 patients (47\%) had AF, 63 patients $(64 \%)$ were males, and the mean age $( \pm \mathrm{SD})$ was $70.8 \pm 10.8$ years old. The period after admission before TEE was $7.9 \pm 4.8$ days. LAT was detected with TEE in 20 patients $(20 \%)$. SEC and LAA slow flow velocity were detected in 41 patients $(42 \%)$ and 50 patients $(51 \%)$, respectively. PFO was found in 23 patients (23\%) and ASA in 19 patients (19\%).

As shown in Table 2, the those factors that were significantly associated with the presence of LAT were male sex (unadjusted odds ratio (OR), 3.94 ; 95\% confidence interval (CI), 1.07-14.58; $p=0.037$ ), presence of AF (unadjusted OR, 9.58 ;
95\% CI, 2.58-35.50; $p<0.001$ ), SEC (unadjusted OR, 8.48 ; 95\% CI, 2.57-28.00; $p<0.001)$ and LAA slow flow velocity (unadjusted OR, $5.18 ; 95 \% \mathrm{CI}$, $1.59-16.91 ; p=0.005)$. In contrast to these factors, age, days from admission to TEE, PFO and

Table 1. Clinical characteristics and TEE findings

\begin{tabular}{cc}
\hline & Total $(n=98)$ \\
\hline Age $( \pm$ SD) & $70.8 \pm 10.8$ \\
Male (\%) & $63(64)$ \\
AF (\%) & $46(47)$ \\
Days from admission to TEE ( \pm SD) & $7.9 \pm 4.8$ \\
Left atrial thrombus (\%) (LAT) & $20(20)$ \\
Spontaneous echo contrast (\%) (SEC) & $41(42)$ \\
left atrial appendage (LAA) & $50(51)$ \\
slow flow velocity (\%) & $23(23)$ \\
Patent foramen ovale (PFO) (\%) & $19(19)$ \\
Atrial septal aneurysm (ASA) (\%) & $9(9)$ \\
PFO+ASA (\%) & $9(9)$ \\
Aortic plaque (\%) & $4(4)$ \\
\hline
\end{tabular}

Table 2. TEE findings associated with left atrial thrombus

\begin{tabular}{cccccr}
\hline & \multicolumn{2}{c}{ LAT } & \multicolumn{3}{c}{ Unadjusted } \\
\hline & $(+) n=20$ & $(-) n=78$ & OR & $95 \%$ CI & $p$-value \\
\hline Age $( \pm \mathrm{SD})$ & $70.5 \pm 10.3$ & $70.9 \pm 11.0$ & - & - & 0.877 \\
Male (\%) & $17(85)$ & $46(59)$ & 3.942 & $1.066-14.576$ & 0.037 \\
AF(\%) & $17(85)$ & $29(37)$ & 9.575 & $2.582-35.502$ & $<0.001$ \\
Days from admission to TEE $( \pm \mathrm{SD})$ & $6.7 \pm 3.7$ & $8.2 \pm 5.0$ & - & - & 0.243 \\
SEC (\%) & $16(80)$ & $25(32)$ & 8.480 & $2.568-27.997$ & $<0.001$ \\
LAA slow flow velocity $(\%)$ & $16(80)$ & $34(44)$ & 5.176 & $1.585-16.906$ & 0.005 \\
PFO (\%) & $3(15)$ & $20(26)$ & 0.512 & $0.136-1.932$ & 0.389 \\
ASA (\%) & $2(10)$ & $17(22)$ & 0.399 & $0.084-1.891$ & 0.346 \\
PFO+ASA (\%) & $1(5)$ & $8(10)$ & 0.461 & $0.054-3.913$ & 0.681
\end{tabular}

LAT, left atrial thrombus ; OR, odds ratio ; $95 \% \mathrm{CI}$, 95\% confidence interval $\mathrm{AF}$, atrial fibrillation ; SEC, spontaneous echo contrast ; LAA, left atrial appendage PFO, patent foramen ovale ; ASA, atrial septal aneurysm.

Table 3. Adjusted odds ratios for TEE findings associated with left atrial thrombus

\begin{tabular}{ccccccc}
\hline & \multicolumn{3}{c}{ Adjusted $^{*}$} & \multicolumn{3}{c}{ Adjusted $^{* *}$} \\
\hline & OR & $95 \%$ CI & $p$-value & OR & $95 \%$ CI & $p$-value \\
\hline Male & 4.429 & $1.134-17.304$ & 0.032 & 5.296 & $1.091-25.714$ & 0.039 \\
AF & 16.153 & $3.171-82.286$ & 0.001 & 8.967 & $1.098-73.196$ & 0.041 \\
SEC & 18.517 & $3.772-90.897$ & $<0.001$ & 10.866 & $1.001-117.999$ & 0.049 \\
LAA slow flow velocity & 8.220 & $2.082-32.456$ & 0.003 & 0.987 & $0.121-8.046$ & 0.991
\end{tabular}

OR, odds ratio ; $95 \% \mathrm{CI}, 95 \%$ confidence interval ; $\mathrm{AF}$, atrial fibrillation

SEC, spontaneous echo contrast ; LAA, left atrial appendage

*adjusted by age and sex

**adjusted by age, sex, days to TEE, AF, and all TEE findings 
ASA were not significantly associated with LAT. Multivariate logistic regression analysis (Table 3) revealed that male sex (adjusted OR, $5.30 ; 95 \% \mathrm{CI}$, $1.09-25.71 ; p=0.039$ ), presence of AF (adjusted OR, 8.97 ; 95\% CI, 1.10-73.20; $p=0.041$ ) and SEC (adjusted OR, 10.87; 95\% CI, 1.001-118.0; $p=$ $0.049)$ were independently associated with LAT, but LAA slow flow velocity was not.

\section{DISCUSSION}

It is well known that TEE is one of the most useful tools and the gold standard for detecting potential sources of embolism from the heart and aorta ${ }^{6}$. In our TEE findings, LAA slow flow velocity was the most common one, SEC second and PFO third. Few all-inclusive reports of TEE findings have been published in Japan. Kuriki et al. ${ }^{7}$ demonstrated TEE findings of stroke inpatients in Japan and reported more cases of PFO than ours.

Although patients with AF often have LAT, there were no significant differences in the days from admission to TEE between those with and without LAT. The period from LAT formation to stroke onset could not be evaluated in our study, which might have affected the results.

The unadjusted odds ratios for male sex, presence of AF, SEC and LAA slow flow velocity showed that all of these factors were significantly associated with LAT. In multivariate logistic regression analysis, male sex, presence of AF and SEC were independently associated with LAT, but LAA slow flow velocity was not. Although we could not find the previous report that showed the association between male sex and LAT regardless of the presence of AF, it is unknown why male sex is a risk factor associated with LAT. However, it may be effecting the finding that the males have higher smoking rate and higher incidence of myocardial infarction than females $^{8}$. In previous studies, patients with nonvalvular AF had an LAT rate that ranged from $2.9 \%$ to $22 \%^{9-15)}$. The rates of LAT detected on TEE after cardioembolic brain infarction (CEBI) ranged from $20 \%$ to $39 \%$ 16-18). Previous reports in patients who had $\mathrm{AF}$ with or without $\mathrm{CEBI}$ have emphasized $\mathrm{D}$ dimer ${ }^{9}$, CHADS2 score ${ }^{10)}$, brain natriuretic peptide (BNP) levels ${ }^{16)}$ and SEC in the $\mathrm{LAA}^{19)}$ as predictors of LAT. Our study also demonstrated that SEC was an independent predictor of LAT, regardless of the presence of AF. SEC was found in about $25 \%$ to $60 \%$ of patients with $\mathrm{AF}$, depending on the patient population ${ }^{20-24)}$, and a study has shown that $12 \%$ of patients with SEC were in sinus rhythm ${ }^{25)}$. It was reported that the stroke or other embolic even rate was $12 \%$ / year in patients who had AF with $\mathrm{SEC}^{20)}$. A previous study in AF patients showed that LAA flow velocity was significantly lower in patients with than without LAT ${ }^{26)}$. In conjunction with our findings, the association between LAA slow flow velocity and LAT may be dependent on the presence of AF.

The current study has some limitations, including the small sample size and the omission of other known predictors of LAT, such as D-dimer and BNP. Furthermore, because of the small sample size, we could not analyze the patients with atherothrombotic stroke and cryptogenic stroke separately. However, it is the main limitation of this study that the analysts of TEE data were the same as the persons who performed TEE.

Within these limitations, we conclude that SEC is an important risk factor for LAT in patients suspected of embolic brain infarction, regardless of whether they have $\mathrm{AF}$.

\section{Conflict and interest statement : None}

\section{REFERENCES}

1. Annual Health, Labour and Welfarw Report 20112012 (Ministry of Health, Labour and Welfare, Japan)

2. Sudlow CLM, Warlow CP. Comparable studies of the incidence of stroke and its pathological types : results from an international collaboration. Stroke, 28 : 491-499, 1997.

3. Special report from the National Institute of Neurological Disorders and Stroke : classification of cerebrovascular disease III. Stroke, 21 : 637676, 1990.

4. Kubo M, Kiyohara Y, Ninomiya T, et al. Decreasing incidence of lacunar vs other types of cerebral infarction in a Japanese population. Neurology, 66 : 1539-1544, 2006.

5. Kimura K, Minematsu K, Yamaguchi T. Atrial fibrillation as a predictive factor for severe stroke and early death in 15,831 patients with acute ischaemic stroke. J Neurol Neurosurg Psychiatry, 76 : 679-683, 2005.

6. de Bruijn SF, Agema WR, Lammers GJ, et al. Transesophageal echocardiography is superior to transthoracic echocardiography in management of patients of any age with transient ischemic attack or stroke. Stroke, 37 : 2531-2534, 2006.

7. Kuriki A, Ichikawa H, Kato H, et al. Transesophageal echocardiography for diagnosis of ischemic stroke subtypes : A hospital-based study of 120 
patients. (in Japanese) Jpn J Stroke, 33:326332, 2011.

8. Ueshima H, Sekikawa A, Miura K, et al. Cardiovascular Disease and Risk Factors in Asia : A Selected Review. Circulation, 118 : 2702-2709, 2008.

9. Habara S, Dote K, Kato M, et al. Prediction of left atrial appendage thrombi in non-valvular atrial fibrillation. Eur Heart J, 28 : 2217-2222, 2007.

10. Wysokinski WE, Ammash N, Sobande F, Kalsi H, Hodge D, McBane RD. Predicting left atrial thrombi in atrial fibrillation. Am Heart J, 159 : 665-671, 2010.

11. Klein AL, Grimm RA, Murray RD, et al. Use of transesophageal echocardiography to guide cardioversion in patients with atrial fibrillation. N Engl J Med, 344 : 1411-1420, 2001.

12. Fukuda S, Shimada K, Kawasaki T, et al. Transnasal transesophageal echocardiography in the detection of left atrial thrombus. J Cardiol, 54 : 425431, 2009.

13. Stoddard MF, Dawkins PR, Prince CR, Ammash NM. Left atrial appendage thrombus is not uncommon in patients with acute atrial fibrillation and a recent embolic event: A transesophageal echocardiographic study. J Am Coll Cardiol, 25 : 452459, 1995.

14. Stoddard MF, Singh P, Dawn B, Longaker RA. Left atrial thrombus predicts transient ischemic attack in patients with atrial fibrillation. Am Heart J, 145 : 676-682, 2003.

15. Yamashita E, Takamatsu H, Tada H, et al. Transesophageal echocardiography for thrombus screening prior to left atrial catheter ablation. Circ J, 74 : 1081-1086, 2010.

16. Okada Y, Shibazaki K, Kimura K, et al. Brain natriuretic peptide is a marker associated with thrombus in stroke patients with atrial fibrillation. J Neurol Sci, 301 : 86-89, 2010.

17. Okuyama H, Hirono O, Tamura H, et al. Usefulness of intensity variation in the left atrial appendage with contrast echocardiography to predict ischemic stroke recurrence in patients with atrial fibrillation. Am J Cardiol, 101 : 1630-1637, 2008.

18. Sadahiro H, Inamura $\mathrm{A}$, Ishihara $\mathrm{H}$, et al. Frag- mental or Massive Embolization in Cardiogenic Stroke Caused by Nonvalvular Atrial Fibrillation. J Stroke Cerebrovasc Dis, 23 : 63-68, 2014.

19. Falcone RA, Morady F, Armstrong WF. Transesophageal echocardiographic evaluation of left atrial appendage function and spontaneous contrast formation after chemical or electrical cardioversion of atrial fibrillation. Am J Cardiol, 78 : 435-439, 1996.

20. Leung DY, Black IW, Cranney GB, Hopkins AP, Walsh WF. Prognostic implications of left atrial spontaneous echo contrast in nonvalvular atrial fibrillation. J Am Coll Cardiol, 24 : 755-762, 1994.

21. González-Torrecilla E, García-Fernández MA, Pérez-David E, Bermejo J, Moreno M, Delcán JL. Predictors of left atrial spontaneous echo contrast and thrombi in patients with mitral stenosis and atrial fibrillation. Am J Cardiol, 86 : 529-534, 2000.

22. Tsai LM, Chen JH, Fang CJ, Lin LJ, Kwan CM. Clinical implications of left atrial spontaneous echo contrast in nonrheumatic atrial fibrillation. Am J Cardiol, 70 : 327-331, 1992.

23. Kamp O, Verhorst PM, Welling RC, Visser CA. Importance of left atrial appendage flow as a predictor of thromboembolic events in patients with atrial fibrillation. Eur Heart J, 20 : 979-985, 1999.

24. Leung DY, Black IW, Cranney GB, et al. Selection of patients for transesophageal echocardiography after stroke and systemic embolic events. Role of transthoracic echocardiography. Stroke, 26 : 1820-1824, 1995.

25. Sadanandan S, Sherrid MV. Clinical and echocardiographic characteristics of left atrial spontaneous echo contrast in sinus rhythm. J Am Coll Cardiol, 35 : 1932-1938, 2000.

26. Jung PH, Mueller M, Schuhmann C, et al. Contrast enhanced transesophageal echocardiography in patients with atrial fibrillation referred to electrical cardioversion improves atrial thrombus detection and may reduce associated thromboembolic events. Cardiovasc Ultrasound, 11 : 1, 2013. doi:10.1186/1476-7120-11-1. 